\title{
Cavity exciton polaritons in two-dimensional semiconductors from first principles
}

\author{
Dino Novko $\odot$ and Vito Despoja $\odot^{*}$ \\ Institute of Physics, Bijenička 46, 10000 Zagreb, Croatia \\ and Donostia International Physics Center (DIPC), P. Manuel de Lardizabal, 4, 20018 San Sebastián, Spain
}

(Received 12 May 2021; revised 12 August 2021; accepted 16 August 2021; published 3 September 2021)

\begin{abstract}
A two-dimensional (2D) semiconducting microcavity, where exciton polaritons can be formed, constitutes a promising setup for exploring and manipulating various regimes of light-matter interaction. Here, the coupling between $2 \mathrm{D}$ excitons and metallic cavity photons is studied by using a first-principles propagator technique. The strength of exciton-photon coupling is characterised by its Rabi splitting to two exciton polaritons, which can be tuned by cavity thickness. Maximum splitting of $128 \mathrm{meV}$ is achieved in the phosporene cavity, while a remarkable value of about $440 \mathrm{meV}$ is predicted in a monolayer hexagonal boron nitride device. The obtained Rabi splittings in a $\mathrm{WS}_{2}$ microcavity are in excellent agreement with recent experiments. The present methodology can aid in predicting and proposing potential setups for trapping robust 2D exciton-polariton condensates.
\end{abstract}

DOI: 10.1103/PhysRevResearch.3.L032056

The interplay between cavity photons and matter can result in the formation of hybrid polarization-photon modes, commonly called polaritons [1]. Due to their dual light-matter nature, these bosonic quasiparticles are enriched with exceptional physical properties, such as small effective mass and nonlinearity, absent in the matter outside the optical cavity. As such, polaritons are expected to show exotic physical phenomena like light-induced superconductivity [2,3], Bose-Einstein condensation [4-6], polariton superfluditiy [7], and quantized vortices [8]. Ideal platforms for studying strong light-matter interactions are gapped systems, such as semincondutors [6,9] or molecules [10], placed in microcavity devices, where exciton polaritons are formed. Cavity exciton polaritons, showing a plethora of quantum effects, are routinely observed in bulk [11-14] and quantum well systems [9,15], e.g., devised from GaAs [9].

Two-dimensional (2D) materials, such as semiconducting monolayers, thin heterostructures and films, are even more attractive than their bulk counterparts, due to the reduced Coulomb screening and the corresponding large exciton binding energies [16-23] that enable formation of well-defined exciton polaritons even at room temperature [24]. The first 2D exciton polaritons were realized in a monolayer of transition metal dichalcogenide (TMD) $\mathrm{MoS}_{2}$, where Rabi splitting between the exciton and a cavity photon of $\sim 50 \mathrm{meV}$ was observed [25]. Further photoluminescence studies showed clear anticrossing behavior and splitting of the exciton polariton in other 2D TMD cavity devices, e.g., in $\mathrm{MoSe}_{2}$ [26], $\mathrm{WS}_{2}$ [27],

\footnotetext{
*vdespoja@ifs.hr

Published by the American Physical Society under the terms of the Creative Commons Attribution 4.0 International license. Further distribution of this work must maintain attribution to the author(s) and the published article's title, journal citation, and DOI.
}

$\mathrm{WSe}_{2}$ [28,29], and in a $\mathrm{MoSe}_{2}-\mathrm{WSe}_{2}$ heterostructure [30]. In addition, real-space imaging of exciton polaritons was done by means of near-field scanning optical microscopy for $\mathrm{WSe}_{2}$ thin films [31]. These TMD semincondutor microcavities are especially appealing due to their charge tunability, coupled spin and valley degrees of freedom, as well as the ability to form heterostructures, and are thus able to display valleypolarized exciton polaritons [32], polaron polaritons [33], and interlayer exciton polaritons [30].

Despite this enormous interest in exciton polaritons and seminconductor microcavity devices, a complementary microscopic theory that is able to scrutinize the cavity photonexciton coupling on the quantitative and predictive level is still rare. In addition, the majority of the microscopic descriptions are based on a simple model Hamiltonians describing exciton-photon interactions in microcavity [34-39]. Recently, a more rigorous $a b$ initio theoretical description of exciton polaritons in a TMD microcavity was provided in the framework of the quantum-electrodynamical Bethe-Salpeter equation [40], where the excitons are calculated from a firstprinciples Bethe-Salpeter equation, and the electromagnetic field is described by quantized photons. However, the coupling between excitons and photons is left to be arbitrary. This study showed how excitonic optical activity and energetic ordering can be controlled via cavity size, light-matter coupling strength, and dielectric environment.

Here, we present a fully quantiative theory of $2 \mathrm{D}$ exciton polaritons embedded in a plasmonic microcavity that is able to analyze and predict light-matter coupling strengths for various cavity settings. We study cavity exciton polaritons in three prototypical two-dimensional single-layer semiconductors, i.e., single-layer black phosphorus or phosphorene $\left(\mathrm{P}_{4}\right)$, $\mathrm{WS}_{2}$, and hexagonal boron nitride (hBN). The results show a clear Rabi splitting between 2D exciton and cavity photon modes as well as a high degree of tunability of Rabi (lightmatter) coupling $\Omega$ as a function of microcavity thickness. In 

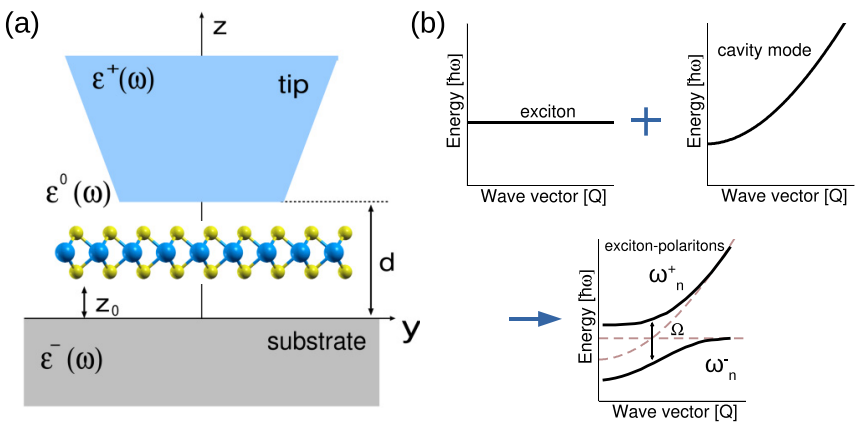

FIG. 1. (a) The schematic of a microcavity device. The 2D crystal described by optical conductivity $\sigma_{\mu}(\omega)$ is inserted in the microcavity which consists of the substrate, the dielectric media and the tip described by the macroscopic dielectric functions $\epsilon^{-}(\omega), \epsilon^{0}(\omega)$ and $\epsilon^{+}(\omega)$, respectively. (b) The intercation between 2D exciton and cavity mode results in creation of exciton polaritons. The coupling strength measure is the Rabi splitting $\Omega$.

the case of $\mathrm{WS}_{2}$, for the usual experimental setup where cavity size is around $d \sim 1 \mu \mathrm{m}$ we obtain splittings of $\Omega \sim 42 \mathrm{meV}$ and $\Omega \sim 64 \mathrm{meV}$ for principal and second cavity modes, in line with the experiments [25,27]. Also, in all of the considered cases larger coupling strengths $\Omega$ are found for larger photon confinements when $d<1 \mu \mathrm{m}$ as well as for higher cavity modes $n$. Interestingly, the ultraviolet (UV) exciton in hBN shows a very strong exciton-cavity photon coupling of about $\sim 440 \mathrm{meV}$ and the possiblity of Bose-Einstein condensation.

In this work both excitons and photons are described by the bosonic propagators $\sigma$ and $\Gamma$, respectively, which are derived from first principles. The 2D crystal optical conductivity $\sigma$ is calculated using the $a b$ initio RPA+ladder method [41], and the propagator of cavity photons $\Gamma$ is derived by solving the Maxwell's equations for planar cavity decribed by local dielectric function $\epsilon$ (see Supplemental Material [42]). The exciton-photon coupling is achieved by dressing the cavityphoton propagator $\Gamma$ with excitons at the RPA level. Thus the obtained results are therefore directly comparable with the experiments. As illustrated in Fig. 1(a) the microcavity device consists of substrate, tip, and dielectric media in between, described by local dielectric functions $\epsilon^{+}, \epsilon^{-}$, and $\epsilon^{0}$, respectively. The 2D semiconducting crystal defined by optical conductivity $\sigma_{\mu}(\omega)$ is immersed in a dielectric media at a height $z_{0}$ relative to the substrate. The substrate occupy region $z<0$, tip occupy region $z>d$, and dielectric media occupy region $0<z<d$. In such a semiconducting microcavity setup the coupling between the exciton and cavity photon is expected to result in the splitting of exciton polariton to the lower and upper polariton branches, which we shall refer to as $\omega_{n}^{-}$and $\omega_{n}^{+}$, respectively [see Fig. 1(b)].

The quantity from which we extract the information about the electromagnatic modes in microcavity setup is electrical field propagator $\mathcal{E}_{\mu \nu}$ which, by definition [43], propagates the electrical field produced by point oscillating dipole $\mathbf{p}_{0} e^{-i \omega t}$, i.e., $\mathbf{E}(\omega)=\mathcal{E}(\omega) \mathbf{p}^{0}$. Assuming that the $2 \mathrm{D}$ crystal, substrate and tip satisfy planar symmetry (in $x-y$ plane) the propagator $\mathcal{E}$ in the $z=z_{0}$ plane satisfies the matrix equation

$$
\mathcal{E}(\mathbf{Q}, \omega)=\boldsymbol{\Gamma}(\mathbf{Q}, \omega)+\boldsymbol{\Gamma}(\mathbf{Q}, \omega) \sigma(\omega) \mathcal{E}(\mathbf{Q}, \omega),
$$

as illustrated by Feynmans diagrams in Fig. 2(a) (see also Sec. S1.A in Ref. [42]). Here $\boldsymbol{\Gamma}=\Gamma^{0}+\Gamma^{s c}$ represents the propagator of electrical field, in the absence of a 2D crystal, i.e., when $\sigma=0$. The propagator $\Gamma^{0}$ represents the "free" electrical field and the propagator of the scattered electrical field $\Gamma^{s c}$ results in multiple reflections at the microcavity interfaces, as ilustrated in Fig. 2(b). In order to simplify the interpretation of the results we suppose that the dielectric media is a vacuum $\left(\epsilon^{0}=1\right)$, and we suppose that the tip and the substrate are made of the same material $\left(\epsilon^{-}=\epsilon^{+}=\epsilon\right)$. In order to support well-defined cavity modes, these materials should be highly reflective in the exciton frequency region $\omega \approx \omega_{e x}$, which is satisfied if $\omega_{e x}<\omega_{p}$, where $\omega_{p}$ is the bulk plasmon frequency. For the $\mathrm{P}_{4}$ and $\mathrm{WS}_{2}$ monolayers where exciton energies are $\hbar \omega_{e x}<3.0 \mathrm{eV}$, we chose that the substrate and tip are made of silver $\left(\omega_{p} \approx 3.6 \mathrm{eV}\right)$. On the other hand, for the single-layer hBN where exciton energy is $\hbar \omega_{e x}=5.67 \mathrm{eV}$ we chose aluminium $\left(\omega_{p} \approx\right.$ $15 \mathrm{eV})$. Both silver and aluminium macroscopic dielectric functions $\epsilon(\omega)$ are determined as well from first principles (see Ref. [42]). As an example, the intensity of electromagnatic modes $\left(-\operatorname{Im} \Gamma_{x}\right)$ in a silver microcavity is shown in Fig. S2 in Ref. [42]. Figure 2(c) shows the perturbative expansion of optical conductivity $\sigma_{\mu}(\omega)=\sigma_{\mu}^{R P A}(\omega)+\sigma_{\mu}^{\text {ladd }}(\omega)$, where $\sigma^{R P A}$ is the RPA optical conductivity [44,45], while the ladder optical conductivity is $\sigma^{\text {ladd }}=\frac{i}{\omega S} j \mathcal{K} j^{*}$, where $S$ is a normalization surface and $j$ are the current vertices. The 4-points polarizability $\mathcal{K}$ satisfies the Dyson equation $[41,42]$ in which the Bethe-Salpeter-Fock kernel $\Phi^{F}$ enters [46-50]. The electron energies are corrected using the GW method so that the present approach is equivalent to the time-dependent screened Hartree-Fock approximation [46-51]. For more details see Secs. S1.B and S2.B in Ref. [42]. Without cavity photons, the total optical absorption $\operatorname{Re} \sigma_{x}(\omega)$ in $\mathrm{P}_{4}$ and $\mathrm{hBN}$ is characterized with the strong excitons appearing at $\hbar \omega_{e x}=1.45 \mathrm{eV}$ and $\hbar \omega_{e x}=5.67 \mathrm{eV}$, respectively. In addition, the absorption spectrum $\operatorname{Re} \sigma_{x}(\omega)$ in $\mathrm{WS}_{2}$ shows the two spin-orbit splitted A and B excitons at $\hbar \omega_{e x}^{A}=2.1 \mathrm{eV}$ and $\hbar \omega_{e x}^{B}=2.46 \mathrm{eV}[42]$.

Figures 3(a), 3(b), and 3(c) show the modifications of the $n=1$ cavity mode intensity after the single-layer $\mathrm{P}_{4}$ is inserted in the middle $z_{0}=d / 2(n=1$ antinodal plane) of the silver cavity, where the cavity sizes are $d=375 \mathrm{~nm}, d=$ $400 \mathrm{~nm}$ and $d=425 \mathrm{~nm}$, respectively. White and turquoise dotted lines denote the $\mathrm{P}_{4}$ exciton and unperturbed cavity mode $n=1$, respectively. For $d=375 \mathrm{~nm}$, just before the $n=1$ cavity mode crosses the exciton, a significant part of the $n=1$ mode spectral weight is transferred below the exciton energy. By increasing the cavity size, i.e., for $d=$ $400 \mathrm{~nm}$ and $d=425 \mathrm{~nm}$, the exciton crosses the $n=1$ mode, which results in the intensity weakening and band-gap opening in the intersection area. This behavior enables creation of an exciton-polariton condensate, as experimentally verified in Refs. [6,13-15,29]. By changing the cavity thickness, the exciton can interact also with the higher cavity modes. Figure 3(d) shows the modifications of $n=2$ mode intensity, where the cavity thickness is $d=850 \mathrm{~nm}$ and $\mathrm{P}_{4}$ is chosen to be located at $z_{0}=d / 4 \mathrm{~nm}$ ( $n=2$ antinodal plane). The exciton significantly weakens the intensity of $n=2$ mode in the intersection area, however, here the avoided crossing 
(a)

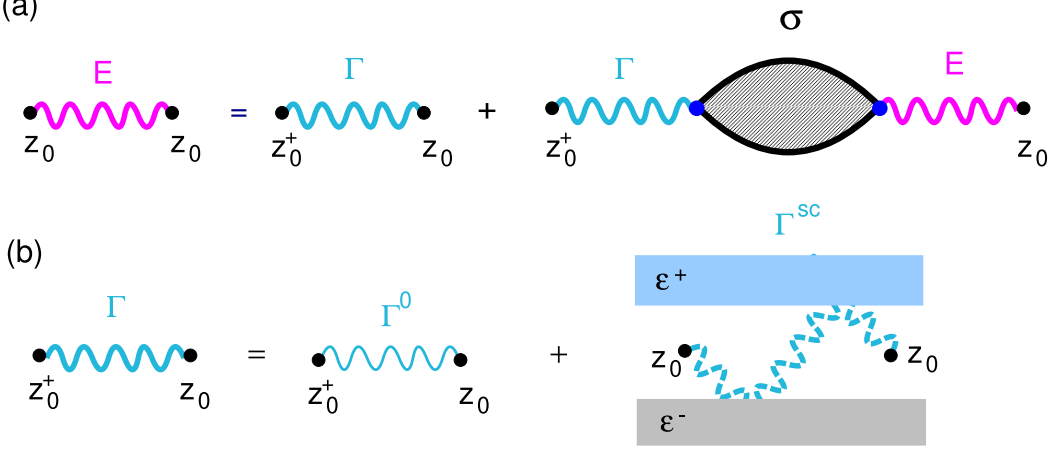

(c)

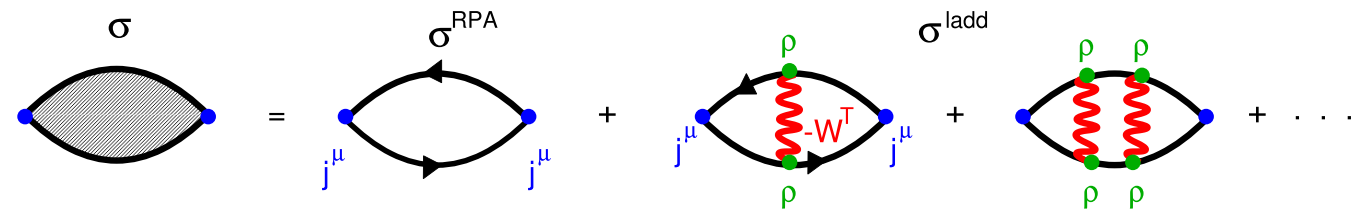

FIG. 2. (a) Dyson's equation for the propagator of the electrical field $\mathcal{E}$ in a microcavity device. (b) Propagator of the electrical field $\Gamma=\Gamma^{0}+\Gamma^{s c}$, in the absence of a $2 \mathrm{D}$ crystal (i.e., when $\sigma_{\mu}=0$ ). (c) Perturbative expansion of optical conductivity $\sigma_{\mu}(\omega)$. Blue dots represent the current vertices $j^{\mu}$, green dots represent the charge vertices $\rho$, and red wavy lines represent the screened Coulomb interaction $W^{T}$.

behavior is not clearly noticeable in comparison with the exciton coupled to the first cavity mode.

The dispersion relation of exciton polaritons $\omega_{n}^{-}$and $\omega_{n}^{+}$ (hybridized cavity photon-exciton modes), as the one shown in Fig. 4(a), can be precisely determined by following the splitted maxima in induced current $j_{\mu}=\sigma_{\mu}^{s c r} E_{\mu}$ driven by external (bare) field $E_{\mu} e^{-i \omega t}$, where the screened optical conductivity is $\sigma_{\mu}^{s c r}=[1-\Gamma \sigma]_{\mu}^{-1} \sigma_{\mu}$. The inset of Fig. 4(b) shows the $\operatorname{Re} \sigma_{x}^{s c r}$ before (brown dashed) and after (solid magenta) $\mathrm{P}_{4}$ is inserted in the middle of the cavity of thickness $d=400 \mathrm{~nm}$. The spliting of exciton $\hbar \omega_{e x}$ to exciton polaritons $\omega_{1}^{-}$and $\omega_{1}^{+}$can be clearly seen. The exciton-photon binding strength can be determined from the Rabi splitting defined as difference $\Omega_{n}=\omega_{n}^{+}-\omega_{n}^{-}$for wave vector $\mathbf{Q}$ and for which the bare cavity modes $n=1,2,3, \ldots$ crosses the exciton $\hbar \omega_{e x}$. Figure 4(a) shows the dispersion relations of plasmon polaritons $\omega_{1}^{-}$and $\omega_{1}^{+}$obtained by following the splitted maxima in $\operatorname{Re} \sigma_{x}^{s c r}$ for different wave vectors $Q_{x}, d=400 \mathrm{~nm}$ and $z_{0}=d / 2$. The clear anticrossing behavior and Rabi splitting of $\Omega_{1}=123 \mathrm{meV}$ indicates a strong interaction between the exciton and $n=1$ cavity photon. Red circles, yellow squares, and green triangles in Fig. 4(b) show the Rabi splittings $\Omega_{n}$ for $n=1, n=2$ and $n=3$, respectively, versus cavity thickness $d$. The maximum Rabi splittings of $n$th mode $\Omega_{n}^{\max }$ are achieved when $z_{0}=d / 2$ and for $d$ chosen so that $n$th mode just starts to cross the exciton energy $\hbar \omega_{e x}$. All three modes show strong coupling with the exciton that results in the maximum splitings of $\Omega_{1}^{\max }=128 \mathrm{meV}, \Omega_{2}^{\max }=92 \mathrm{meV}$ and $\Omega_{3}^{\max }=77 \mathrm{meV}$ for $d=390 \mathrm{~nm}, d=820 \mathrm{~nm}$ and $d=$ $1250 \mathrm{~nm}$, respectively. For larger $d$ the cavity modes cross the exciton at a greater angle and the splitting decreases. A decrease of $\Omega_{n}^{\max }$ as $n$ increases confirms a confinement hypothesis; as $n$ increases the cavity photon modes crosses the exciton for larger $d$, and photon becomes less confined while the coupling is reduced. The confinement hypothesis is in accordance with the predictions of the two boson Hamiltonian model, where the Rabi splitting is proportional to the square root of the exciton oscillator strength multiplied by the
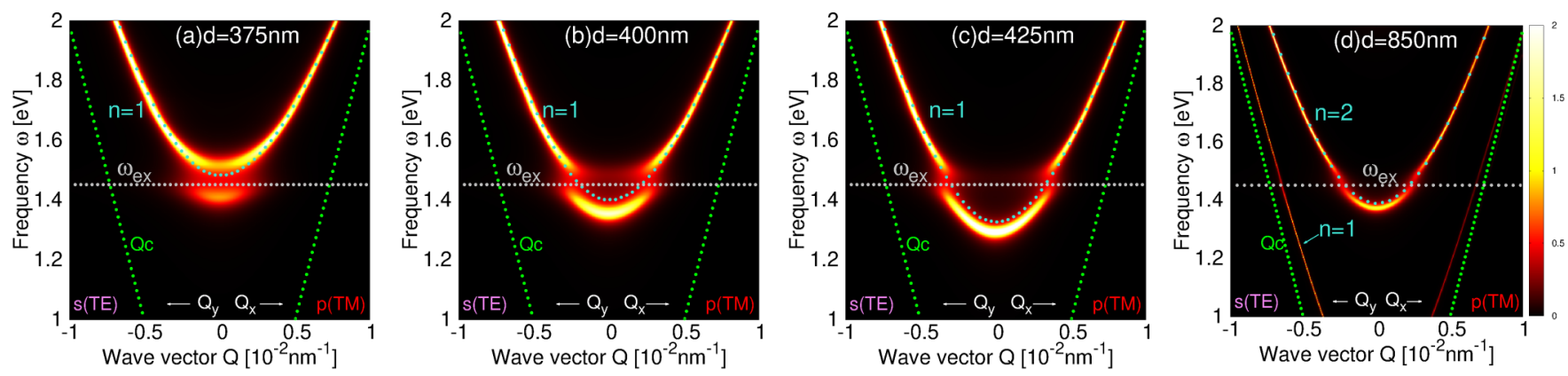

FIG. 3. The intensities of electromagnatic modes $\left(-\operatorname{Im} \mathcal{E}_{x x}\right)$ showing the hybiridization between silver cavity mode $n=1$ (turquoise dotted) and $\mathrm{P}_{4}$ exciton (white dotted) for various cavity sizes: (a) $d=375 \mathrm{~nm}$, (b) $d=400 \mathrm{~nm}$, and (c) $d=425 \mathrm{~nm}$, where $z_{0}=d / 2$. (d) The hybridization between cavity mode $n=2$ and $\mathrm{P}_{4}$ exciton in cavity of thickness $d=850 \mathrm{~nm}$ and $z_{0}=d / 4$. 

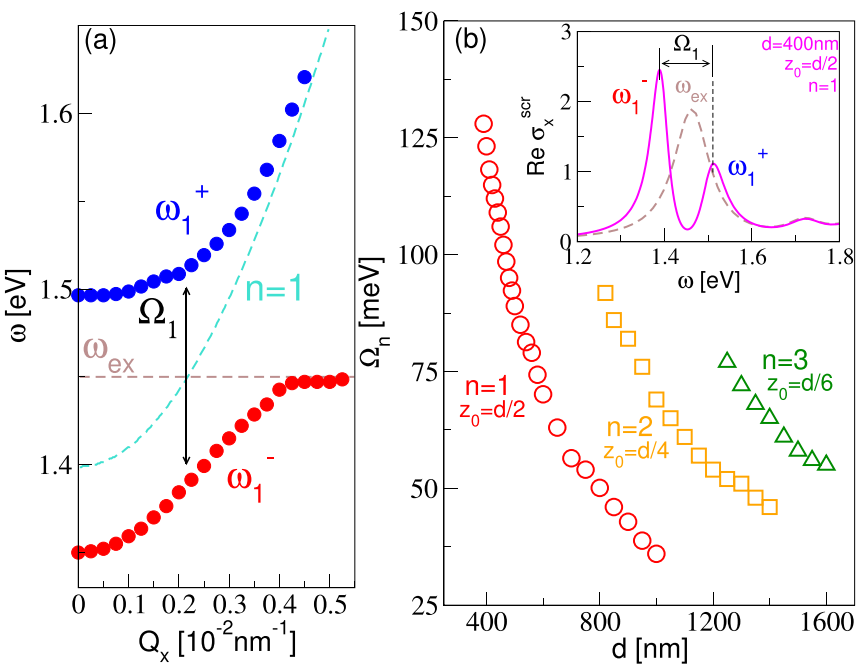

FIG. 4. (a) The dispersion relations of exciton polaritons $\omega_{1}^{-}$and $\omega_{1}^{+}$(dots) for $\mathrm{P}_{4}$ in silver microcavity of thickness $d=400 \mathrm{~nm}$ and $z_{0}=d / 2$. Dashed lines show unperturbed modes. (b) The Rabi splittings $\Omega_{n}=\omega_{n}^{+}-\omega_{n}^{-}$versus cavity thickness $d$ for $n=1$ (red circles), $n=2$ (orange squares) and $n=3$ (green triangles) cavity modes, where $z_{0}=d / 2, z_{0}=d / 4$ and $z_{0}=d / 6$, respectively. The inset shows $\operatorname{Re} \sigma_{x}^{s c r}$ before (brown dashed) and after (solid magenta) the $\mathrm{P}_{4}$ is inserted in the cavity, where $d=400 \mathrm{~nm}, z_{0}=d / 2$ and $Q_{x}=0.00217 \mathrm{~nm}^{-1}$ (momentum for which exciton crosses the $n=1$ mode).

overlap between exciton and cavity-photon wave functions [52]. Moreover, due to this fact the Rabi splitting is proportional to the square root of the microcavity mode frequency $\Omega_{1} \sim \sqrt{\omega_{1}^{0}(Q=0)}$. Considering that in the perfect reflectivity limit $\left(r_{s, p} \approx \mp 1\right)$ the cavity modes disperses as [42]

$$
\omega_{n}^{0}(Q)=c \sqrt{\frac{n^{2} \pi^{2}}{d^{2}}+Q^{2}}, \quad n=1,2,3, \ldots
$$

we can estimate the Rabi splitting as $\Omega_{1} \sim 1 / d$. The overlap between the exciton and the cavity-photon wave functions is enhanced if the $2 \mathrm{D}$ crystal is put in the middle of the cavity $\left(z_{0}=d / 2\right)$, where the cavity-photon wave function has a maximum. The mentioned effect on the Rabi splitting is a pure geometric effect. However, it is important to note that the
Rabi splitting is also defined by the quantum effects, which are stored in exciton-photon coupling functions (Eq. (S19) in Ref. [42]). These are the exciton intensity (exciton oscillatory strength) and a measure of the symmetry overlap between the cavity photon and exciton, e.g., defined by the dipole selection rule for the principal mode $\omega_{1}^{0}$. Thus, the coupling will be stronger as the thickness $d$ at which the crossing between $n$th mode and exciton occurs is getting smaller, and, equally important, when the exciton oscillatory strength is larger. The importance of both the geometric and quantum effects is demonstrated later when comparing the $\mathrm{WS}_{2}$ and $\mathrm{P}_{4}$ results.

The above criterion is met by excitons with higher excitation energy, such as, for instance, the UV exciton in the $\mathrm{hBN}$ single layer. Since in the same UV frequency region the cavity should be highly reflective (i.e., $\omega_{p}>\omega_{e x}$ ), the appropraite cavity for $\mathrm{hBN}$ layer can be made of aluminium with $\omega_{p} \approx 15 \mathrm{eV}$. Figure 5(a) shows the modification of aluminium $n=1$ cavity mode intensity after the hBN monolayer is inserted in the middle of cavity of thicknesses $d=90 \mathrm{~nm}$. The dotted lines show the unperturbed cavity mode $n=1$ and the $\mathrm{hBN}$ exciton. The strong exciton-photon intercation results in strong modification of the $n=1$ cavity mode, band-gap opening and Rabi splitting of $\Omega_{1}=400 \mathrm{meV}$. The maximum Rabi splitting of $\Omega_{1}^{\max }=440 \mathrm{meV}$ is achieved for $d=85 \mathrm{~nm}$. This large exciton-polariton band gap and Rabi splitting suggests a possibility of experimental realization of robust excitonpolariton condensate in the cavity setup. It should be noted that the hBN Rabi splitting is here relatively large (relative to the other 2D crystals), however, the absolute splitting, relative to the cavity-photon energy $\omega_{1}^{0}(Q=0) \sim 1 / d$, does not seem so extraordinary. Despite that, the strong hBN exciton oscillatory strength makes the absolute splitting large. In fact, even for very small $d$ below $100 \mathrm{~nm}$, such a large splitting would not be possible in the two other 2D materials considered here, due to the energy of the corresponding exciton peaks and weaker oscillatory strengths.

The 2D exciton-polaritons are experimentally studied mostly in various TMDs where the mesured Rabi splittings of $\Omega=46 \mathrm{meV}, \Omega=26 \mathrm{meV}$, and $\Omega=20 \mathrm{meV}$ are found in $\mathrm{MoS}_{2}$ [25], $\mathrm{WSe}_{2}$ [28], and $\mathrm{MoSe}_{2}$ [26]. For $\mathrm{WS}_{2}$ the experimentally measured splittings are around $20-70 \mathrm{meV}$ for $d>1 \mu \mathrm{m}$, depending on the precise cavity size [27]. In Fig. 5(b) we show the modification of the silver $n=1$ cavity mode intensity when the $\mathrm{WS}_{2}$ monolayer is inserted
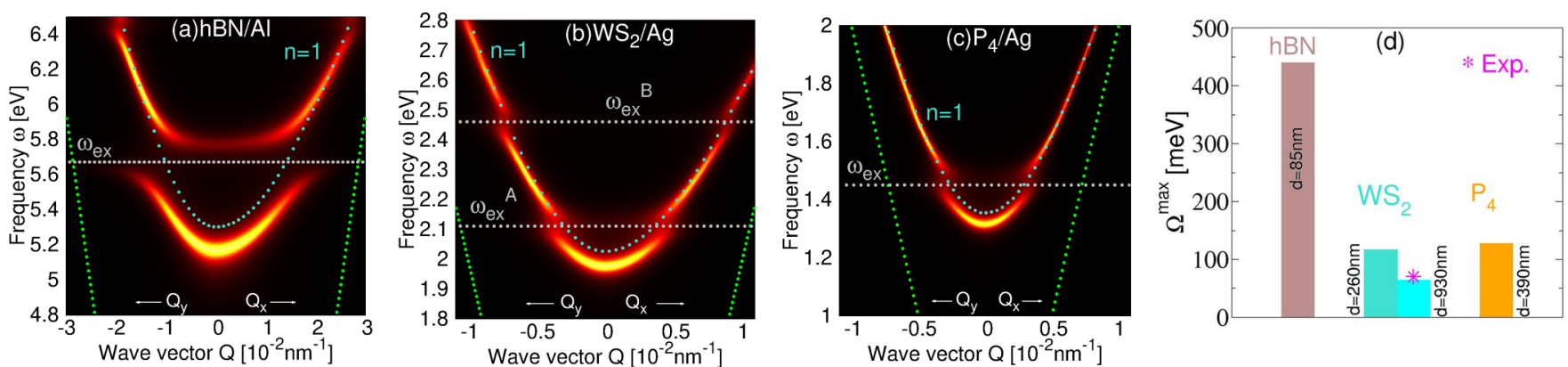

FIG. 5. The intensity of electromagnatic modes $\left(-\operatorname{Im} \mathcal{E}_{x x}\right)$ showing the hybridization between cavity mode $n=1$ (turquoise dotted) and (a) hBN exciton (white dotted) in aluminium cavity of thickness $d=90 \mathrm{~nm}$, (b) the $\mathrm{WS}_{2} \mathrm{~A}$ and B excitons in silver cavity of thickness $d=260 \mathrm{~nm}$, and (c) the $\mathrm{P}_{4}$ exciton in silver cavity of thickness $d=415 \mathrm{~nm}$. The $z_{0}=d / 2$ in all cases. (d) Summary of the obtained Rabi splittings in studied systems. The experimental value for $\mathrm{WS}_{2}$ microcavity is from Ref. [27]. 
in the middle of the microcavity of thickness $d=260 \mathrm{~nm}$. The unperturbed $n=1$ mode as well as the $\mathrm{A}$ and $\mathrm{B}$ excitons of bare $\mathrm{WS}_{2}$ are also denoted by dotted lines. Both excitons significantly perturb the $n=1$ mode providing the Rabi splitings of $\Omega_{1}^{A}=117 \mathrm{meV}$ and $\Omega_{1}^{B}=103 \mathrm{meV}$. For comparison, Fig. 5(c) shows the modification of $n=1$ mode intensity when $\mathrm{P}_{4}$ is inserted in silver microcavity for the same conditions as in $\mathrm{WS}_{2}$ microcavity presented in Fig. 5(b); $n=1$ minimum is $100 \mathrm{meV}$ below the exciton. The cavity thickness is $d=415 \mathrm{~nm}$ and $z_{0}=d / 2$. Interestingly, the achieved Rabi splitting is here also $\Omega_{1}=117 \mathrm{meV}$, even though, according to the confinement hypothesis, the A exciton, which is confined in a smaller cavity, is expected to split more. However, the A exciton in $\mathrm{WS}_{2}$ has smaller oscillatory strength than $\mathrm{P}_{4}$ exciton [cf. Figs. S4(a) and S5(b) in Ref. [42]] so that the binding is weaker and the two effects, i.e., geometric and quantum, compete.

Finally, in Fig. 5(d) we compare the maximum splittings of exciton-polaritons $\Omega^{\max }$ for the three semiconducting microcavities, summarizing the different regimes of excitoncavity photon coupling strengths in these materials. For $\mathrm{WS}_{2}$ microcavity we additionally present the results for the experimentally measured value of cavity size, i.e., $d=930 \mathrm{~nm}$ [27], when the exciton interacts with $n=2$ cavity mode. The corresponding value of $\Omega_{2}^{A}=64 \mathrm{meV}$ shows an excellent agreement with the experiment [27]. For the same cavity thickness, splitting of the $n=1$ cavity mode and the $\mathrm{WS}_{2} \mathrm{~A}$ exciton is $\Omega_{1}^{A}=42 \mathrm{meV}$.

In summary, we have studied the interaction strengths between cavity photons and excitons in representative and relevant 2D semiconducting crystals by means of rigorous $a b$ initio methodology. It is shown that insertion of $2 \mathrm{D}$ crystals into a metallic microcavity significantly modifies the photon dispersion. For instance, the band gap opening and Rabi splitting as high as $\Omega=440 \mathrm{meV}$ was obtained for $\mathrm{hBN}$ cavity device. This opens the possibility of experimental realization of the robust $2 \mathrm{D}$ exciton-polariton condensate. Moreover, the exciton-photon interaction strongly depends on photon confinement, which was shown to be adjustable by the cavity thickness $d$. The results of exciton-polariton splitting in the $\mathrm{WS}_{2}$ cavity device show a good agreement with recent experiments and suggests higher photon confinements with decreasing cavity size at which stronger photon-matter coupling should be achieved. In order to reach this stronger binding we suggest an experimental setup consisting of tunable submicrometer cavity (such as Atomic-ForceMicroscope tip and substrate) tuned so that the principal photon cavity mode coincide with the exciton energy, e.g., as in Fig. 4(b).

The authors acknowledge financial support from European Regional Development Fund for the "Center of Excellence for Advanced Materials and Sensing Devices" (Grant No. KK.01.1.1.01.0001) and "QuantiXLie Centre of Excellence" (Grant KK.01.1.1.01.0004), as well as from Croatian Science Foundation (Grant No. IP-2020-02-5556 and Grant No. UIP2019-04-6869). Computational resources were provided by the Donostia International Physic Center (DIPC) computing center.
[1] D. N. Basov, Ana Asenjo-Garcia, P. James Schuck, X. Zhu and A. Rubio, Polariton panorama, Nanophotonics 10, 549 (2021).

[2] F. Schlawin, A. Cavalleri, and D. Jaksch, Cavity-Mediated Electron-Photon Superconductivity, Phys. Rev. Lett. 122, 133602 (2019).

[3] J. B. Curtis, Z. M. Raines, A. A. Allocca, M. Hafezi, and V. M. Galitski, Cavity Quantum Eliashberg Enhancement of Superconductivity, Phys. Rev. Lett. 122, 167002 (2019).

[4] J. Kasprzak, M. Richard, S. Kundermann et al., Bose-Einstein condensation of exciton polaritons, Nature (London) 443, 409 (2006).

[5] R. Balili, V. Hartwell, D. Snoke, L. Pfeiffer, and K. West, BoseEinstein Condensation of Microcavity Polaritons in a Trap, Science 316, 1007 (2007).

[6] H. Deng, H. Haug, and Y. Yamamoto, Exciton-polariton BoseEinstein condensation, Rev. Mod. Phys. 82, 1489 (2010).

[7] A. Amo, J. Lefrère, S. Pigeon et al., Superfluidity of polaritons in semiconductor microcavities, Nat. Phys. 5, 805 (2009).

[8] K. G. Lagoudakis, M. Wouters, M. Richard et al., Quantized vortices in an exciton-polariton condensate, Nat. Phys. 4, 706 (2008).

[9] C. Weisbuch, M. Nishioka, A. Ishikawa, and Y. Arakawa, Observation of the Coupled Exciton-Photon Mode Splitting in a Semiconductor Quantum Microcavity, Phys. Rev. Lett. 69, 3314 (1992).

[10] R. F. Ribeiro, L. A. Martínez-Martínez, M. Du, J. CamposGonzalez-Angulo, and J. Yuen-Zhou, Polariton chemistry: controlling molecular dynamics with optical cavities, Chem. Sci. 9, 6325 (2018).

[11] S. Christopoulos, G. Baldassarri Höger von Högersthal, A. J. D. Grundy et al., Room-Temperature Polariton Lasing in Semiconductor Microcavities, Phys. Rev. Lett. 98, 126405 (2007).

[12] J. J. Baumberg, A. V. Kavokin, S. Christopoulos et al., Spontaneous Polarization Buildup in a Room-Temperature Polariton Laser, Phys. Rev. Lett. 101, 136409 (2008).

[13] S. Zhang, J. Chen, J. Shi et al., Trapped Exciton-Polariton Condensate by Spatial Confinement in a Perovskite Microcavity, ACS Photonics 7, 327 (2020).

[14] W. Bao, X. Liu, F. Xue et al., Observation of Rydberg exciton polaritons and their condensate in a perovskite cavity, Proc. Natl. Acad. Sci. USA 116, 20274 (2019).

[15] T. Horikiri, T. Byrnes, K. Kusudo, N. Ishida, Y. Matsuo, Y. Shikano, A. Löffler, S. Höfling, A. Forchel, and Y. Yamamoto, Highly excited exciton-polariton condensates, Phys. Rev B 95, 245122 (2017).

[16] A. Ramasubramaniam, Large excitonic effects in monolayers of molybdenum and tungsten dichalcogenides, Phys. Rev. B 86, 115409 (2012).

[17] Y. Li, A. Chernikov, X. Zhang, A. Rigosi, H. M. Hill, A. M. van der Zande, D. A. Chenet, En-Min Shih, J. Hone, and T. F. Heinz, Measurement of the optical dielectric function of monolayer transition-metal dichalcogenides: $\mathrm{MoS}_{2}, \mathrm{MoSe}_{2}, \mathrm{WS}_{2}$, and $\mathrm{WSe}_{2}$, Phys. Rev. B 90, 205422 (2014). 
[18] D. Y. Qiu, F. H. Jornada, and S. G. Louie, Optical Spectrum of $\mathrm{MoS}_{2}$ : Many-Body Effects and Diversity of Exciton States, Phys. Rev. Lett. 111, 216805 (2013).

[19] Y. Lin, X. Ling, L. Yu, S. Huang, Allen L. Hsu, Yi-Hsien Lee, J. Kong, M. S. Dresselhaus, and T. Palacios, Dielectric Screening of Excitons and Trions in Single-Layer $\mathrm{MoS}_{2}$, Nano Lett. 14, 5569 (2014).

[20] J. Yan, K. W. Jacobsen, and K. S. Thygesen, Optical properties of bulk semiconductors and graphene/boron nitride: The Bethe-Salpeter equation with derivative discontinuity-corrected density functional energies, Phys. Rev. B 86, 045208 (2012).

[21] F. Ferreira and R. M. Ribeiro, Improvements in the GW and Bethe-Salpeter-equation calculations on phosphorene, Phys. Rev B 96, 115431 (2017).

[22] C. E. P. Villegas, A. S. Rodin, A. C. Carvalho, and A. R. Rocha, Two-dimensional exciton properties in monolayer semiconducting phosphorus allotropes, Phys. Chem. Chem. Phys. 18, 27829 (2016).

[23] X. Wang, A. M. Jones, K. L. Seyler, V. Tran, Y. Jia, H. Zhao, Han Wang, Li Yang, X. Xu, and F. Xia, Highly anisotropic and robust excitons in monolayer black phosphorus, Nat. Nanotechnol. 10, 517 (2015).

[24] T. Low, A. Chaves, J. D. Caldwell, A. Kumar, N. X. Fang, P. Avouris, T. F. Heinz, F. Guinea, L. Martin-Moreno, and Frank Koppens, Polaritons in layered two-dimensional materials, Nat. Mater. 16, 182 (2017).

[25] Xiaoze Liu, Tal Galfsky, Zheng Sun, Fengnian Xia, E.-C. Lin, Y.-H. Lee, S. Kéna-Cohen, and Vinod M. Menon, Strong light-matter coupling in two-dimensional atomic crystals, Nat. Photonics 9, 30 (2015).

[26] S. Dufferwiel, S. Schwarz, F. Withers, A. A. P. Trichet, F. Li, M. Sich, O. Del Pozo-Zamudio, C. Clark, A. Nalitov, D. D. Solnyshkov, G. Malpuech, K. S. Novoselov, J. M. Smith, M. S. Skolnick, D. N. Krizhanovskii, and A. I. Tartakovskii, Exciton-polaritons in van der Waals heterostructures embedded in tunable microcavities, Nat. Commun. 6, 8579 (2015).

[27] L. C. Flatten, Z. He, D. M. Coles, A. A. P. Trichet, A. W. Powell, R. A. Taylor, J. H. Warner, and J. M. Smith, Roomtemperature exciton-polaritons with two-dimensional $\mathrm{WS}_{2}$, Sci. Rep. 6, 33134 (2016)

[28] S. Dufferwiel, T. P. Lyons, D. D. Solnyshkov, A. A. P. Trichet, A. Catanzaro, F. Withers, G. Malpuech, J. M. Smith, K. S. Novoselov, M. S. Skolnick, D. N. Krizhanovskii, and A. I. Tartakovskii, Valley coherent exciton-polaritons in a monolayer semiconductor, Nat. Commun. 9, 4797 (2018).

[29] Jie Gu, V. Walther, L. Waldecker, D. Rhodes, A. Raja, J. C. Hone, T. F. Heinz, S. Kéna-Cohen, T. Pohl, and V. M. Menon, Enhanced nonlinear interaction of polaritons via excitonic Rydberg states in monolayer $\mathrm{WSe}_{2}$, Nat. Commun. 12, 2269 (2021).

[30] M. Förg, L. Colombier, R. K. Patel, J. Lindlau, A. D. Mohite, H. Yamaguchi, M. M. Glazov, D. Hunger, and A. Högele, Cavitycontrol of interlayer excitons in van der Waals heterostructures, Nat. Commun. 10, 3697 (2019).

[31] Z. Fei, M. E. Scott, D. J. Gosztola, J. J. Foley IV, J. Yan, D. G. Mandrus, H. Wen, P. Zhou, D. W. Zhang, Y. Sun, J. R. Guest, S. K. Gray, W. Bao, G. P. Wiederrecht, and X. Xu, Nano-optical imaging of $\mathrm{WSe}_{2}$ waveguide modes revealing light-exciton interactions, Phys. Rev. B 94, 081402(R) (2016).
[32] Z. Sun, J. Gu, A. Ghazaryan et al., Optical control of room-temperature valley polaritons, Nat. Photonics 11, 491 (2017).

[33] M. Sidler, P. Back, O. Cotlet et al., Fermi polaron-polaritons in charge-tunable atomically thin semiconductors, Nat. Phys. 13, 255 (2017).

[34] D. S. Citrin and J. B. Khurgin, Microcavity effect on the electron-hole relative motion in semiconductor quantum wells, Phys. Rev. B 68, 205325 (2003).

[35] J. Levinsen, Guangyao Li, and Meera M. Parish, Microscopic description of exciton-polaritons in microcavities, Phys. Rev. Research 1, 033120 (2019).

[36] F. Xue, Fengcheng Wu, Ming Xie, Jung-Jung Su, and A. H. MacDonald, Microscopic theory of equilibrium polariton condensates, Phys. Rev. B 94, 235302 (2016).

[37] Y. N. Gartstein, Xiao Li, and C. Zhang, Exciton polaritons in transition-metal dichalcogenides and their direct excitation via energy transfer, Phys. Rev. B 92, 075445 (2015).

[38] J. B. Khurgin, Two-dimensional exciton-polariton-light guiding by transition metal dichalcogenide monolayers, Optica 2, 740 (2015).

[39] A. Krasnok, S. Lepeshov, and A. Alú, Nanophotonics with 2D transition metal dichalcogenides, Opt. Express 26, 15972 (2018).

[40] S. Latini, E. Ronca, U. De Giovannini, H. Hübener, and A Rubio, Cavity Control of Excitons in Two-Dimensional Materials, Nano Lett. 19, 3473 (2019).

[41] D. Novko, K. Lyon, D. J. Mowbray, and V. Despoja, Ab initio study of electromagnetic modes in two-dimensional semiconductors: Application to doped phosphorene, arXiv:2106.05583.

[42] See Supplemental Material at http://link.aps.org/supplemental/ 10.1103/PhysRevResearch.3.L032056 which additionally includes Refs. [53-68], for more details on calculation of electrical field propagator and optical conductivity, silver dielectric function, spectrum of bare silver cavity, optical absorption spectra of phosphorene, $\mathrm{hBN}$, and $\mathrm{WS}_{2}$ monolayers with excitonic effects included, as well as on computational details.

[43] M. S. Tomaš, Green function for multilayers: Light scattering in planar cavities, Phys. Rev. A 51, 2545 (1995).

[44] V. Despoja, M. Šunjić, and L. Marušić, Propagators and spectra of surface polaritons in metallic slabs: Effects of quantum-mechanical nonlocality, Phys. Rev. B 80, 075410 (2009).

[45] D. Novko, M. Šunjić, and V. Despoja, Optical absorption and conductivity in quasi-two-dimensional crystals from firs principles: Application to graphene, Phys. Rev B 93, 125413 (2016).

[46] L. Hedin, New method for calculating the one-particle Green's function with application to the electron-gas problem, Phys. Rev. 139, A796 (1965).

[47] G. Strinati, Effects of dynamical screening on resonances at inner-shell thresholds in semiconductors, Phys. Rev. B 29, 5718 (1984).

[48] G. Onida, L. Reining, and A. Rubio, Electronic excitations: density-functional versus many-body Green's-function approaches, Rev. Mod. Phys. 74, 601 (2002).

[49] Mark S. Hybertsen and Steven G. Louie, Electron correlation in semiconductors and insulators: Band gaps and quasiparticle energies, Phys. Rev. B 34, 5390 (1986). 
[50] M. Rohlfing and S. G. Louie, Electron-Hole Excitations in Semiconductors and Insulators, Phys. Rev. Lett. 81, 2312 (1998).

[51] W. Hanke and L. J. Sham, Many-Particle Effects in the Optical Excitations of a Semiconductor, Phys. Rev. Lett. 43, 387 (1979).

[52] E. A. Cotta and P. M. S. Roma, Determination of oscillator strength of confined excitons in a semiconductor microcavity, Condens. Matter Phys. 17, 23702 (2014).

[53] W. Hanke and L. J. Sham, Many-particle effects in the optical spectrum of a semiconductor, Phys. Rev. B 21, 4656 (1980).

[54] M. Rohlfing and S. G. Louie, Excitons and Optical Spectrum of the $\mathrm{Si}(111)-(2 \times 1)$ Surface, Phys. Rev. Lett. 83, 856 (1999).

[55] M. Rohlfing and S. G. Louie, Electron-hole excitations and optical spectra from first principles, Phys. Rev. B 62, 4927 (2000).

[56] R. Tian, R. Fei, S. Hu, T. Li, B. Zheng, Y. Shi, J. Zhao, L. Zhang, X. Gan, and X. Wang, Observation of excitonic series in monolayer and few-layer black phosphorus, Phys. Rev. B 101, 235407 (2020).

[57] J. Yang, R. Xu, J. Pei, Ye Win Myint, F. Wang, Z. Wang, S. Zhang, Z. Yu, and Yuerui Lu, Optical tuning of exciton and trion emissions in monolayer phosphorene, Light Sci. Appl. 4, e312 (2015).

[58] Likai Li, J. Kim, C. Jin, G. Jun Ye, D. Y. Qiu, F. H. da Jornada, Z. Shi, L. Chen, Z. Zhang, F. Yang, K. Watanabe, T. Taniguchi, W. Ren, S. G. Louie, X. Hui Chen, Y. Zhang, and Feng Wang, Direct observation of the layer-dependent electronic structure in phosphorene, Nat. Nanotechnol. 12, 21 (2017).

[59] Ziliang Ye, Ting Cao, Kevin O’Brien, Hanyu Zhu, Xiaobo Yin, Yuan Wang, Steven G. Louie, and Xiang Zhang, Probing ex- citonic dark states in single-layer tungsten disulphide, Nature (London) 513, 214 (2014).

[60] B. Zhu, X. Chen, and X. Cui, Exciton binding energy of monolayer $\mathrm{WS}_{2}$, Sci. Rep. 5, 9218 (2015).

[61] P. Giannozzi, S. Baroni, N. Bonini, M. Calandra, R. Car, C. Cavazzoni, D. Ceresoli, G. L. Chiarotti, M. Cococcioni, I. Dabo et al., QUANTUM ESPRESSO: a modular and open-source software project for quantum simulations of materials, J. Phys.: Condens. Matter 21, 395502 (2009).

[62] N. Troullier and J. L. Martins, Efficient pseudopotentials for plane-wave calculations, Phys. Rev. B 43, 1993 (1991).

[63] D. R. Hamann, Optimized norm-conserving Vanderbilt pseudopotentials, Phys. Rev. B 88, 085117 (2013).

[64] J. P. Perdew, K. Burke, and M. Ernzerhof, Generalized Gradient Approximation Made Simple, Phys. Rev. Lett. 77, 3865 (1996).

[65] J. P. Perdew and A. Zunger, Self-interaction correction to density-functional approximations for many-electron systems, Phys. Rev. B 23, 5048 (1981).

[66] H. J. Monkhorst and J. D. Pack, Special points for Brillouinzone integrations, Phys. Rev. B 13, 5188 (1976).

[67] J. J. Mortensen, L. B. Hansen, and K. W. Jacobsen, Real-space grid implementation of the projector augmented wave method, Phys. Rev. B 71, 035109 (2005).

[68] J. Enkovaara, C. Rostgaard, J. J. Mortensen, J. Chen, M. Dulak, L. Ferrighi, J. Gavnholt, C. Glinsvad, V. Haikola, H. A. Hansen, H. H. Kristoffersen, M. Kuisma, A. H. Larsen, L. Lehtovaara, M. Ljungberg, O. Lopez-Acevedo, P. G. Moses, J. Ojanen, T. Olsen, V. Petzold, N. A. Romero et al., Electronic structure calculations with GPAW: a real-space implementation of the projector augmented-wave method, J. Phys.: Condens. Matter 22, 253202 (2010). 